\title{
The Application Research on Ultrasonic Partial Discharge Detection for Transformer
}

\author{
Yanpeng $\mathrm{Li}^{\mathrm{a}}$, Tianzheng Wang ${ }^{\mathrm{b}}$, Jichong Liang ${ }^{\mathrm{c}}$, Zhumao $\mathrm{Lu}{ }^{\mathrm{d}}$, \\ Yongxin Liu ${ }^{\mathrm{e}}$, Dongdong Yang ${ }^{\mathrm{f}}$, Lu Bai ${ }^{\mathrm{g}}$ and Liqiang $\mathrm{Ma}^{\mathrm{h}}$ \\ State Grid Shanxi Electric Power Research Institute, Taiyuan 030001, China; \\ aliyanpeng1982@163.com, bwtz2000@163.com, cchonggen@163.com, dluzhumao@163.com, \\ e18935129186@163.com, ‘55444809@qq.com, 918bailu@163.com, h253388071@qq.com
}

Keywords: Transformer, Ultrasonic, Partial discharge (PD), PD source location.

\begin{abstract}
As an effective discharged detection technology, the ultrasonic partial discharge detection can discover the potential defects of power equipment in operation, which has been applied in the fault diagnosis to the GIS, transformer, cable connector and the high voltage switchgear, etc. But in the practical application, due to the problems such as interference exclusion, signal identification and positioning, it is difficult to the property and location of defect to make accurate judgments. In this paper, based on ultrasonic discharge test case for transformer with defects, the ultrasonic discharge diagnosis method of transformer defect are analyzed and summarized, and the characteristics of the transformer ultrasonic partial discharge is proposed.
\end{abstract}

\section{Introduction}

When the partial discharge occurs in operating transformer, this phenomenon accompanied such as electrical pulse, ultrasonic, light, heating and chemical change etc. The ultrasonic signal in the form of spherical wave is transmitted form through the dielectric such as insulating cardboard and insulating oil to transformer tank at some speed [1-3] .

The transformer ultrasonic discharge testing judge whether the transformer exist partial discharge by the detection and the analysis of ultrasonic signal along with PD. The ultrasonic signal is obtained by the ultrasonic sensors which is placed on the transformer tank surface.

But the transformer internal structure is complex, including winding, insulation dielectric, oil, magnetic shielding etc, the ultrasonic suffers from attenuation though the working medium. Especially when the ultrasonic transmit from one dielectric to the other, causes interfacial attenuation due to many differences in the acoustic character impedance. The greater the impedance difference, the greater the decay.

The partial discharge fault in outer winding occurs, the acoustic signal can generally spread to the tank and has good coupling with sensor, and the majority of ultrasonic signals can be detected. It is difficult to detect the internal winding partial discharge because of the winding and oil attenuation.

The ultrasonic signal from the signal source will be transmitted to the sensor via multiple paths. The direct propagation path is that acoustic signal transmit to ultrasonic sensor in a straight line. The other way is the composite propagation path, which is transmitted to the tank near the signal source, and then through the tank wall to the ultrasonic sensor. In the ultrasonic PD testing, the general selection is the signal transmitted directly to the ultrasonic sensor, ignoring the smaller ultrasonic signals coming from the composite path.

After confirming that the PD signal is transformer inner, the position of the PD signal source can be positioned in two ways by the electro-acoustical or acoustical-acoustical time delay.

Due to the complexity of the transformer internal structure, the propagation velocity, path and attenuation of ultrasound in a variety of media is complex and changeable. It is difficult to pinpoint the ultrasonic source position, but it is possible to determine the area of the PD source through the judgment and analysis to ultrasonic signal, further provide useful help for transformer defect location. 


\section{Application and Research of Ultrasonic detection}

\subsection{On-site Application}

In a $220 \mathrm{kV}$ operating substation, a transformer has an abnormal discharge signal by ultrasonic discharge testing. The ultrasonic sensor frequency range is $35-300 \mathrm{khz}$, and the instrument adopts four fixed ultrasonic probes in order to locate the calculation. In addition, the instrument also configures a high frequency current transformer, which is used to obtain an electric PD signal. Detecting location can be triggered by electrical or acoustic signal.

The high frequency signal form the core earth wire of the transformer is as follows:

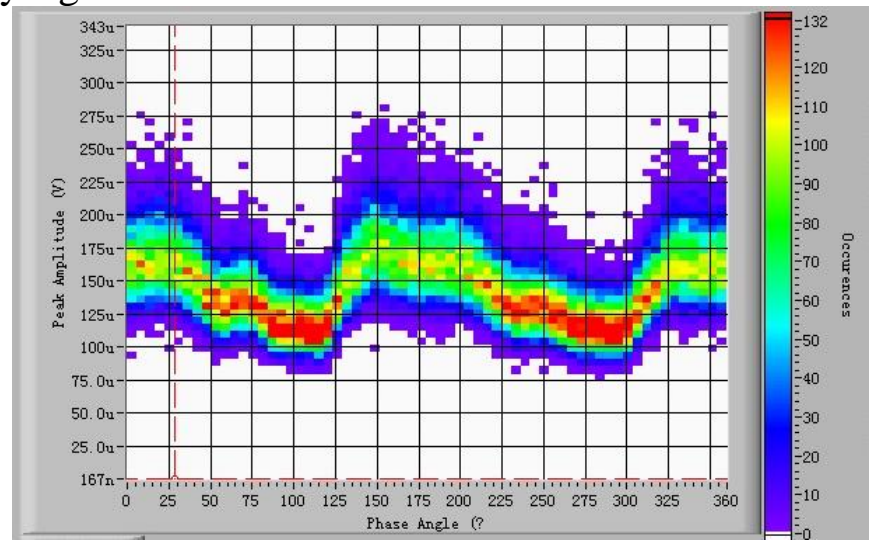

Fig.1 The PRPD graph of PD signal

PRPD graph shows transformer exist two partial discharge in 20ms, Based on the PD characteristics, we can determine that there is suspended discharge or surface discharge.

After detecting the abnormal signal, it is should be located by point-by-point detecting ultrasonic signal and probing on the transformer oil tank.

The obvious acoustic signal is detected when the ultrasonic probe is decorated in transformer tank location under B phase medium-voltage bushing turret. After repeated measurements for many times, the similar signals can be detected all the time. The sensor moves in position and finds that the suspected signal has shrunk or disappeared. The four sensors ultrasonic signal graph is as follows:

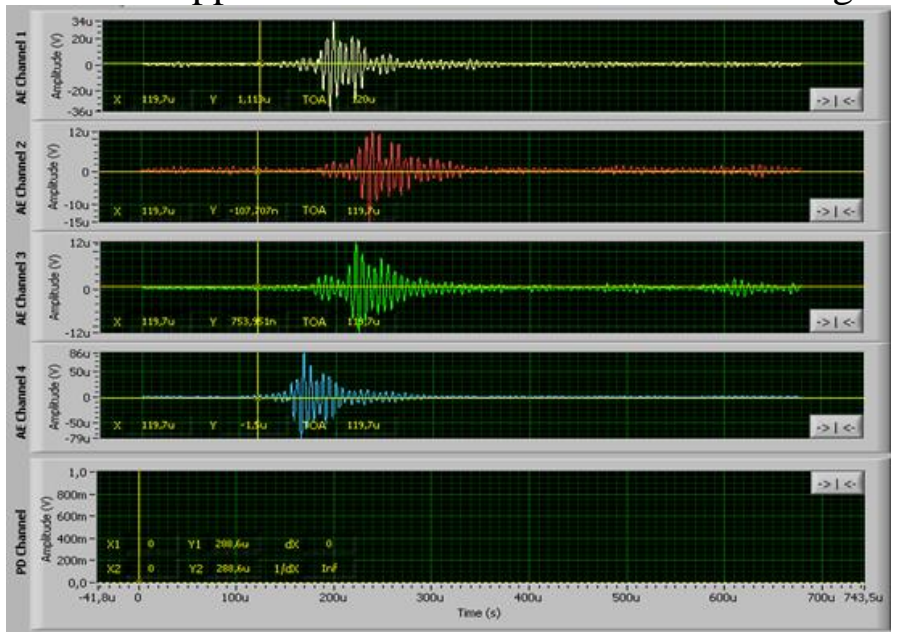

Fig. 2 The ultrasonic signal graph

After the acquisition of ultrasonic signal data, the frequency-domain characteristics of $1,3,4$ channel signal data is obtained by the spectral analysis method, the amplitude frequency characteristics graph is shown as figure 3 . 


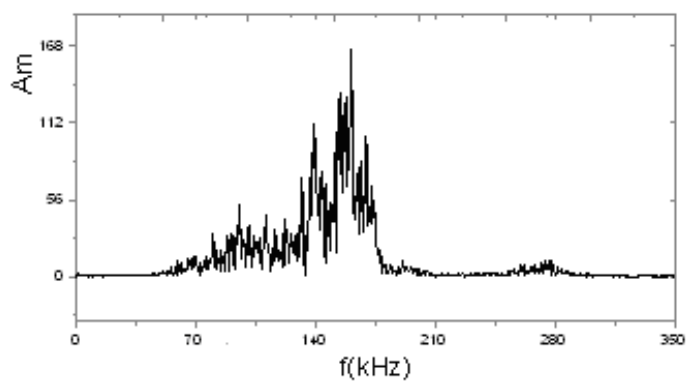

a) The graph of first channel

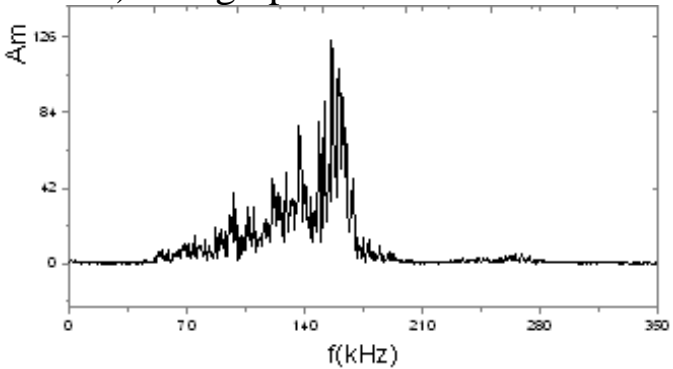

b) The graph of second channel

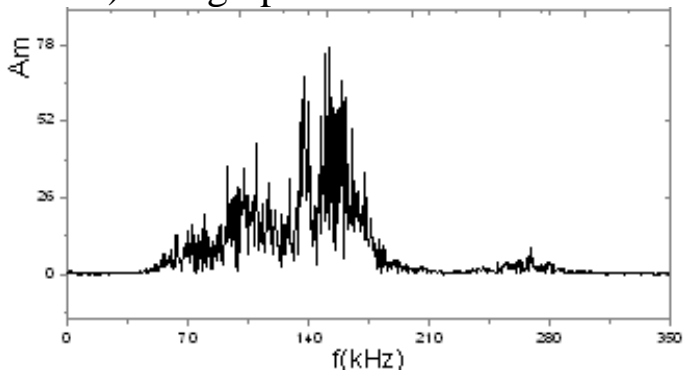

c) The graph of third channel

Fig. 3 The frequency spectrum of ultrasonic signal

The results from the frequency spectrum analysis shows three channels ultrasonic signal frequency band is consistent and is form the same signal source. The frequency band is $70-180 \mathrm{kHz}$, and conforms to the partial discharge frequency band. The ultrasonic spectrum range of the transformer vibration noise generally is less than $30 \mathrm{kHz}$ [4]

Determine transformer is internal partial discharge signals, the four channels ultrasonic signals are used to the positioning calculation analysis of signal source. The four ultrasonic sensors are decorated on the transformer oil tank walls, by using ultrasound time lag to different sensor, the space position of signal source is calculated. Because ultrasonic wave propagation through a variety of media such as copper, oiled paper, in order to convenient calculation, the equivalent wave velocity is used to replace real propagation velocity of sound waves in the transformer, usually take $1400 \mathrm{~m} / \mathrm{s}$, and then through the sound velocity and time delay to calculate and locate coordinate interval of PD source.

Positioning calculation results shows that the PD source is the direction of the medium-voltage bushing, as shown in the figure 4 . The yellow thick line in figure shows the location direction of partial discharge source, the cubic lattice shows transformer outline, the four green points shows location of four ultrasonic probe.

Based on the filed ultrasonic signal, the ultrasonic attenuation characteristics and the positioning analysis result, it is concluded that PD source area is the interval form B phase medium-voltage bushing to bushing lifting pedestal. Then, dissolved gas component content in insulating oil was analyzed, and the result shows acetylene content was changed from 0 to $10.5 \mathrm{ppm}$, which also further proved that there was partial discharge inside the transformer. 


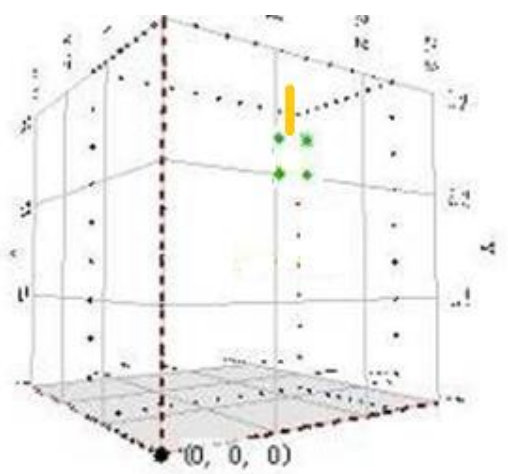

Fig.4 The PD source location three-dimensional graph

\subsection{Disintegration verification}

Combined with field transformer ultrasonic PD positioning result, it is taken to focus on the position form B phase medium-voltage bushing to bushing lifting pedestal. It is found that there is carbon black formed PD on medium-wire clamp inside lifting pedestal, as shown in the figure 5.

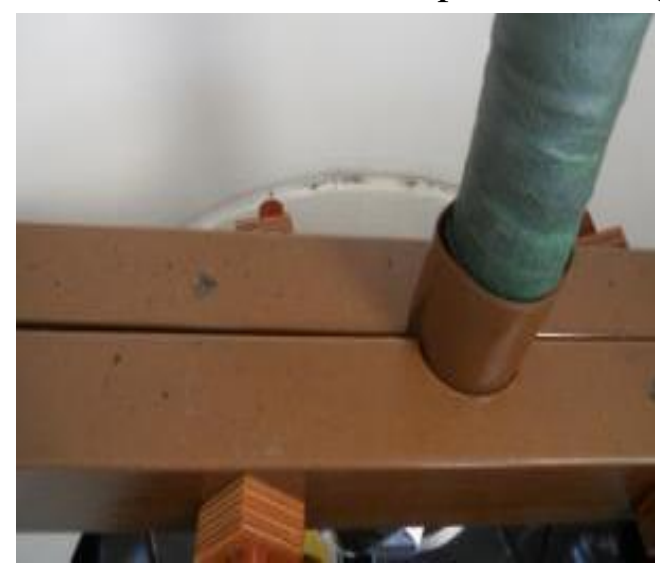

Fig.5 Carbon black formed PD

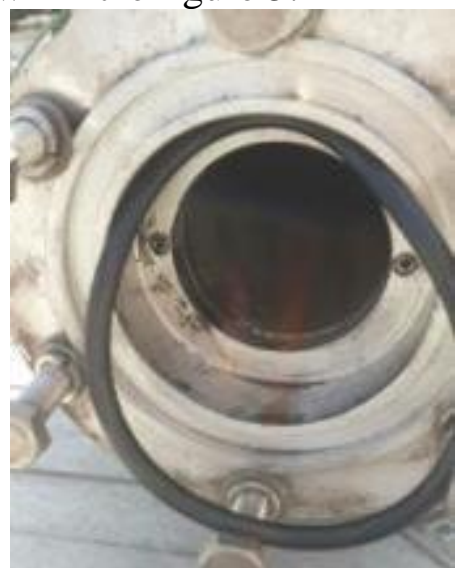

Fig.6 Discharge source location graph

Based on the discharge formation position and the cable through type bushing structure, it is shown that PD source is come from inside bushing. Further examination found that discharge position is the lead wire joint of the phase B medium-voltage bushing, the discharge reason is internal joint lead poor contact to form the suspended discharge, as shown in figure 6.

\section{Conclusion}

The transformer ultrasonic partial discharge detection is able to detect internal discharge within the operating condition of transformer. Especially in the high voltage leading wire of transformer, the ultrasonic signals are more easily measured in these parts.

When the suspected signal is detected, it should be analyzed from the repeatability, spectrum, phase characteristic, amplitude and duration of the ultrasonic signal. The frequency of the ultrasonic signals in the transformer is typically $40-300 \mathrm{khz}$, the amplitude is typically $40-80 \mathrm{db}$, the discharge is associated with $50 \mathrm{~Hz}$, and there should be a certain time delay between the different channel acquighting signals.

After arranging the ultrasonic probe, for every channel, the trigger threshold value and the signal gain should be set according to the background noise. Make sure that each channel's signal has the obvious rising edge to locate the signal source.

\section{References}

[1].Junhao Li, Wenrong Si,Song Wang,et al.Situation and development of PD location method for power transformer.Transformer.Vol.44(2007)No.6,p.40-44. 
[2].Fuhr J,Hassig M,Boss P,et ai.Detection and location of internal defects in the insulation of power transformers.IEEE Trans.on EI,Vol.28(1993)No.6,p.1057-1067.

[3].Lixing Zhou,Lei He,Weiguo Li.Research on Characteristics of Ultrasonic Signal of Partial Discharge and Partial discharge Sources Location In Transformer.High Voltage Engineering. Vol.29 (2003)No.5,p.11-13,16.

[4].Zhirong Wu,Huanzhang Li,Liang Tang.Analysis of the Frequency Spectra of Acoustic Signals in On-line Power Transformer and Reactor.High Voltage Engineering.(1991)No.1,p.46-50. 